\title{
Membranes with different hydration levels: the interface between bound and unbound hydration water.
}

\author{
Carles Calero, Giancarlo Franzese \\ Secció de Física Estadística i Interdisciplinària-Departament de Física de la Matèria \\ Condensada, Facultat de Física $\mathbb{E}$ Institute of Nanoscience and Nanotechnology (IN2UB), \\ Universitat de Barcelona, Martí i Franquès 1, 08028 Barcelona, Spain
}

\begin{abstract}
The interaction of water with membranes is fundamental in many biological processes. Recently we found that, upon increasing hydration, water molecules first fill completely the interior of the membrane, next accumulate in layers in the exterior region. Here, we show by all-atom simulations that the translational and rotational dynamics of water molecules is strongly determined by their local distance to the membrane so that we can identify the existence of an interface between the first hydration shell, partially made of hydration water bound to the membrane, and the next shells entirely made of unbound hydration water. Bound hydration water has a possible structural role and an extremely slow dynamics, while unbound hydration water, with no water-lipids hydrogen bonds, has a dynamics ten time faster than bound water but still one order of magnitude slower than bulk water. Our results could be relevant to understand the slowdown of biological activity upon dehydration.

Keywords: water; phospholipid membrane; molecular dynamics; confinement; translational diffusion; rotational dynamics; structure; biological interfaces.
\end{abstract} Franzese)

Email addresses: carles.calero@ub.edu (Carles Calero), gfranzese@ub.edu (Giancarlo Franzese) 


\section{Introduction}

The structure and function of biological membranes is greatly determined by the properties of hydration water. In fact, the stability and fluidity of the phospholipid bilayer-the main component of biological membranes-is a consequence of the hydrophobic effect, which favours a reduction of the exposure of the apolar hydrocarbon tails of phospholipids to water. Interfacial water also plays an important role in the tasks that the cell membrane performs, mainly related to transport and signalling functions, since it mediates the interaction between membranes and solutes such as ions, proteins, DNA and other membranes [1, 2. For these reasons it is essential to have a proper description of the structural and dynamical properties of water at the interface with membranes.

Phospholipid bilayers or monolayers of a single type of phospholipid are used as model systems to understand the basic properties of more complex biological membranes, which also contain proteins, sugars and cholesterol in large proportions. In particular, the properties of hydration water at phospholipid membranes have been extensively investigated in experiment [3, 4, 5, 6, 7, 8, 9, 10] and using computer simulations [11, 12, 2, 13, 14, 15, 16. NMR spectroscopy has been used to study the translational dynamics of interfacial water, evidencing the different rates of lateral and normal diffusion and revealing the effect of lipid hydration on water dynamics [3, 4, The slowdown of water dynamics due to the interaction with the phospholipid membrane has also been observed with the help of molecular dynamics (MD) simulations [12, 2]. NMR experiments and vibrational sum frequency generation spectroscopy have provided insight on the ordering and orientation of water molecules around phospholipid headgroups [5, 6, in agreement with the picture extracted from computer simulations of hydrated phospholipid membranes [11, 2]. Infrared spectroscopy measurements indicate the formation of strong hydrogen bonds (HBs) with the phosphate and carbonyl groups of phospholipids, as well as an enhancement of the HBs between water molecules in the vicinity of phospholipid headgroups [7, 6]. The rotational dynamics of water molecules is also dramatically affected 
by the presence of phospholipids and the hydration level of the membrane, as evidenced experimentally using a variety of techniques including ultrafast vibrational spectroscopy [8, terahertz spectroscopy [9], and neutron scattering [10]. MD simulations have complemented these studies by exploring the decay of water orientation correlation functions in phospholipid membranes with different hydration levels [13, 14, 15, 16].

The studies mentioned above indicate that the dynamics and structure of interfacial water is determined by the proximity to the phospholipid membrane and by its level of hydration. In view of this evidence, after a short summary of our previous results, here we investigate using all-atom MD simulations the structure and dynamics of hydration water as a function of both the water local distance to a dimyristoylphosphatidylcholine (DMPC) phosholipid membrane and the level of hydration of the membrane. With this approach we identify the water layers relevant to understand the overall translational and rotational dynamics of hydration water. Among a wide variety of lipids, DMPC are phospholipids incorporating a choline as a headgroup and a tailgroup formed by two myristoyl chains. Choline based phospholipids are ubiquitous in cell membranes and used in drug targeting liposomes [1.

\section{Methods}

\subsection{Simulations}

As in Ref. [15, the simulated system consists of a bilayer membrane of 128 DMPC lipids distributed in two leaflets in contact with hydration water (Fig. 1). We consider phospholipid bilayers with six different hydration levels (i.e., water molecules per lipid) $\omega=4,7,10,15,20$, and 34 . This range extends from the weakly hydrated systems probed in recent experiments $8,9,17,10$, to a fully hydrated membrane (with hydration level $\omega=34$ ), which has been thoroughly studied both experimentally and using computer simulations [18, 12.

We perform MD simulations using the NAMD 2.9 [19] package at a temperature of $303 \mathrm{~K}$ and an average pressure of $1 \mathrm{~atm}$. We set the simulation time step 


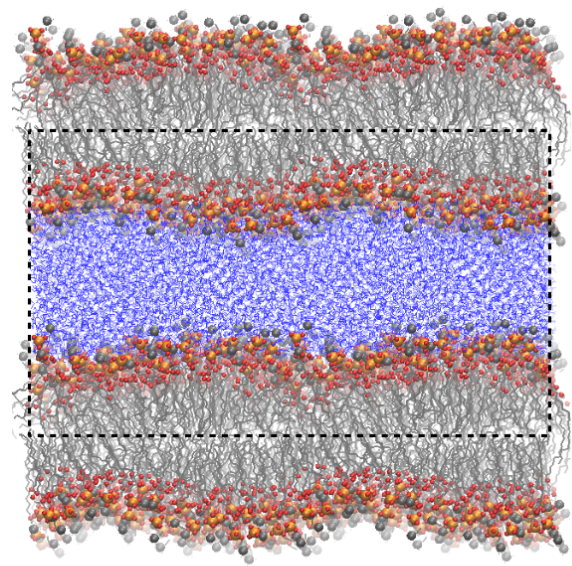

Figure 1: Snapshot of a representative case of the systems considered in our study with hydration level $\omega=34$. The beads are color coded to represent different elements (gray: nitrogen, red: oxygen, orange: phosphorous). Gray lines represent the hydrophobic tails of the phospholipid and blue lines water molecules. The dashed line indicates the size of the simulation box. By convention, we set the vertical dashed lines parallel to the $z$ axis, and the horizontal dashed line to the $x$ axis of the reference frame. The simulated system has a typical area of $\simeq 3900 \AA^{2}$ and a height from $\simeq 38 \AA$ for $\omega=4$ to $\simeq 69 \AA$ for $\omega=34$. 
to $1 \mathrm{fs}$. We describe the structure of phospholipids and their mutual interactions using the recently parameterized force field CHARMM36 [20, 21], which is able to reproduce the area per lipid in excellent agreement with experimental data. The water model employed in our simulations, consistent with the parametrization of CHARMM36, is the modified TIP3P [22, 23]. We cut off the Van der Waals interactions at $12 \AA$ with a smooth switching function starting at $10 \AA$. We compute the long-range electrostatic forces using the particle mesh Ewald method [24] with a grid space of $\simeq 1 \AA$. We update the electrostatic interactions every 2 fs. After energy minimization, we equilibrate the hydrated phospholipid bilayers for $10 \mathrm{~ns}$ followed by a production run of $50 \mathrm{~ns}$ in the NPT ensemble at $1 \mathrm{~atm}$.

We use a Langevin thermostat [25] with a damping coefficient of $0.1 \mathrm{ps}^{-1}$ to control the temperature and a Nosé-Hoover Langevin barostat [26] with a piston oscillation time of $200 \mathrm{fs}$ and a damping time of $100 \mathrm{fs}$ to control the pressure. Aksimentiev and Schulten shown that the Langevin thermostat with a small damping coefficient has a negligible effect on dynamical quantities for a system with parameters as in our simulations [27]. Furthermore, the use of a barostat is important in MD simulations of membranes, because constant volume simulations do not allow for sufficient pressure relaxation and the system does not equilibrate to appropriate pressures. This effect has been reported in the literature for systems consisting of phospholipid monolayers [28] and phospholipid bilayers [29] and has its origin in the lack of fluctuations of the periodic cell, which restricts the phospholipids from assuming energetically favorable conformations. On the other hand, the use of periodic boundary conditions in our simulations allows us to describe a system of perfectly stacked phospholipid bilayers with a homogeneous prescribed hydration level $\omega$.

\subsection{Definition of local distance from the interface}

To properly define the interface between water and phospholipid membranes a suitable definition of a distance to the membrane is required. Indeed, the water-membrane interface is not flat when observed at the relevant length-scale 
given by the size of the water molecule: it exhibits spatial inhomogeneities of $\simeq 1 \mathrm{~nm}$. Such inhomogeneities are dynamical, but they change over times significantly longer than the relevant timescale of water dynamics. This is evidenced by the disparity of diffusion coefficients: while the typical diffusion coefficient of water is of the order of $1 \mathrm{~nm}^{2} / \mathrm{ns}$, the diffusion coefficient of phospholipids is of $\simeq 0.001 \mathrm{~nm}^{2} / \mathrm{ns}[30$. In addition, the interface is soft and not easily defined because water molecules can penetrate into the membrane, as shown by experiments 31] and numerical simulations [32, 33. In order to describe the interface, we adopt a local definition of the water molecules distance from the membrane devised by Pandit et al. [32, 2. Given the planar symmetry of our 3-dimensional setup (Fig. 1), the definition is the following: for each snapshot we perform a 2-dimensional Voronoi tesselation of the planar projection of the membrane into the $x y$-plane, using as centers of the cells the phosphorous and nitrogen atoms of the phospholipid heads. To each water molecule we assign the Voronoi cell given by its center of mass projection into the $x y$-plane, and a distance $\xi \equiv z_{\text {water }}-z_{\text {Voronoy }}$ to the membrane given by the difference between the $z$-coordinates of the water molecule, $z_{\text {water }}$, and the $z$-coordinates of the corresponding Voronoi cell, $z_{\text {Voronoy }}$. Smondyrev and Berkowitz shown that the vector connecting phosphorous and nitrogen atoms, when the membrane is solvated by water, is in average almost parallel to the interface [34. Therefore, calculating the local distance including both membrane atoms allows a better definition than just including the phosphorous atoms 32 because it avoids the overestimate of the distance, while the possible systematic error is small [34].

\subsection{Structural observables}

For all the hydration levels, we study the structure of the hydrogen bond (HB) network by considering HBs formed by water with other water molecules or with oxygen atoms in phosphate and carboxylate groups of the DMPC phospholipid. We calculate as function of $\xi$ the water density profile and the average number of $\mathrm{HBs}\left\langle n_{\mathrm{HB}}\right\rangle \equiv\left\langle 2 N_{\mathrm{HB}}\right\rangle / N$, where $N_{\mathrm{HB}}$ and $N$ are the total number of HBs and water molecules in the system, respectively, and $\langle\cdot\rangle$ represents 
the thermodynamics average. The normalized distribution of water-water and water-lipid $\left\langle N_{\mathrm{HB}}\right\rangle$ provides an estimate of the probability of a water molecule to be H-bonded with another moiety at different regions of the interface. For the calculation of $\left\langle N_{\mathrm{HB}}\right\rangle$ we symmetrize the results from the two leaflets of the phospholipid bilayer.

\subsection{Dynamical observables}

We calculate the mean square displacement on the plane of the membrane $\left(\operatorname{MSD}_{\|}(t)\right)$ of the center of mass of water molecules located in layers at different distances $\xi$ from the membrane. In general, particles at a given layer eventually leave and enter other layers which are characterized by different dynamics. Hence, an unrestricted calculation of $\operatorname{MSD}_{\|}(t)$ over all the molecules in the layer $i$ at a given time would mix dynamics of different layers if the times is large-enough. On the other hand, evaluate $\mathrm{MSD}_{\|}(t)$ by selecting only the water molecules which stay in the studied layer during at least the entire time used for the calculation would induce a bias favoring the slow molecules, which are more likely to stay in the layer during the entire time, with respect to the very mobile water molecules.

Therefore, to avoid a significant bias we need, first, to calculate for each layer the average time that a water molecules spends in a layer without leaving it and, second, estimate $\operatorname{MSD}_{\|}(t)$ over this specific time by considering only those water molecules which remain within the considered layer over the entire time interval. The largest time interval over which $\operatorname{MSD}_{\|}(t)$ is well defined is given by the characteristic residence time $\tau_{\text {res }}^{(i)}$ that a water molecule spends in the layer $i$ [35]. To estimate $\tau_{\text {res }}^{(i)}$ we calculate the survival probability $S^{(i)}(\tau)$, which is the probability that a given water molecule stays in the layer $i$ for a time interval $\tau$. We define $S^{(i)}(\tau)$ as 35 .

$$
S^{(i)}(\tau) \equiv\left\langle\frac{N^{(i)}\left(t_{0}+\tau\right)}{N^{(i)}\left(t_{0}\right)}\right\rangle
$$

where $N^{(i)}\left(t_{0}+\tau\right)$ is the number of water molecules remaining in layer $i$ during the time interval $\tau$ and that were part of the number $N^{(i)}\left(t_{0}\right)$ in the same layer 
at $t=t_{0}$. The brackets $\langle\ldots\rangle$ indicate average over different time origins $t_{0}$. We estimate the residence times for the layer $i$ using

$$
\tau_{\text {res }}^{(i)} \equiv \int_{0}^{\infty} S^{(i)}(t) d t
$$

Once we know $\tau_{\text {res }}^{(i)}$, we can characterize the diffusion on the plane of the membrane by calculating the diffusion coefficient for each layer $D_{\|}(\xi)$, within the residence time.

We study also the reorientation dynamics of water molecules at different layers by calculating the rotational dipolar correlation function,

$$
C_{\mathrm{sim}}^{\mathrm{rot}}(t) \equiv\langle\hat{\mu}(t) \cdot \hat{\mu}(0)\rangle
$$

where $\hat{\mu}(t)$ is the direction of the water dipole vector at time $t$ and $\langle\ldots\rangle$ denote ensemble average over all water molecules and time origins.

Finally, we calculate the HB time correlation functions

$$
C_{\mathrm{HB}}^{\mathrm{w}-\alpha}(t) \equiv \frac{\left\langle n^{\mathrm{w}-\alpha}(t) n^{\mathrm{w}-\alpha}(0)\right\rangle}{\left\langle n^{\mathrm{w}-\alpha}(0)\right\rangle},
$$

where the index $n^{\mathrm{w}-\alpha}(t)=1$ when at time $t$ a given water forms a HB with another water $(\alpha=\mathrm{w})$ or a lipid $(\alpha=1)$, and is zero otherwise. The brackets $\langle\ldots\rangle$ indicate averaging over all water-water or water-lipid group pairs and multiple time origins. $C_{\mathrm{HB}}^{\mathrm{w}-\alpha}(t)$ provides a measure of the probability that a $\mathrm{HB}$ at time 0 remains formed at a later time $t$.

\section{Results and Discussion}

\subsection{Structure: bound and unbound hydration water}

As in Ref. [15, we calculate the density of water molecules as a function of the local distance to the membrane $\xi$ for the phospholipid bilayers with different hydration level (Fig 2). From inspection of the water density profile for the fully hydrated membrane (with hydration level $=34$ ) we can clearly observe that it displays a local maxima at $\xi<0$ and another at $\xi_{\min }>33 \AA$ just before vanishing at the largest distance. These two maxima correspond to the water molecules 


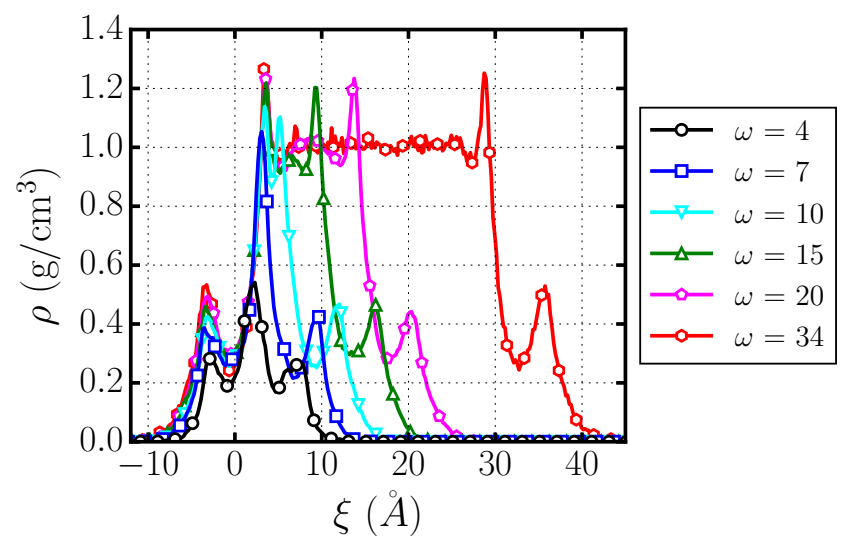

Figure 2: Water density profile for stacked DMPC phospholipid bilayers at different hydration levels: $\omega=4$ (black dots), $\omega=7$ (blue squares), $\omega=10$ (cyan down triangles), $\omega=15$ (green up triangles), $\omega=20$ (magenta pentagons), and $\omega=34$ (red hexagons). Symbols are placed to identify the corresponding lines. In all the cases, the average $x y$-plane of one of the two membrane layers is at $\xi=0$. Water molecules at (i) $\xi<0$ are within the reference membrane layer, (ii) at $0<\xi<\xi_{\min }$ are outside the two membrane layers, with the average $x y$-plane of the second membrane layer at $\xi_{\min }$ where the largest minimum of the density profile occurs (e.g., $\xi_{\min } \simeq 33 \AA$ for $\omega=34$ ), and (iii) at $\xi>\xi_{\min }$ are within the second membrane layer. For high hydration, e.g., $\omega=34$, within the region (ii) a clear maximum is observable at $\approx 5 \AA$ from each membrane layer, representing the first hydration layer $(0<\xi<5 \AA$ and $28 \AA$ $<\xi<\xi_{\min } \simeq 33 \AA$ ). The water in the range $5 \AA<\xi<28 \AA$ is exterior to the two stacked membranes. 
within the interior of each DMPC phosholipid leaflet. The minima at $\xi=0$ and $\xi_{\min } \simeq 33 \AA$ are the average $x y$-planes of the two membrane layers. In the range $0<\xi<\xi_{\min }$ we can distinguish three main regions: the first hydration layer of the first leaflet $(0<\xi<5 \AA)$, exterior layers $(5 \AA<\xi<28 \AA)$, and the first hydration layer of the second leaflet $\left(28 \AA<\xi<\xi_{\min } \simeq 33 \AA\right)$. For the less hydrated cases the same structure is preserved, although the exterior region is thinner (in the cases with $\omega=15,20$ ) or nonexistent (in the cases with $\omega=4$, $7,10)$.

We observe that, as hydration increases, water molecules accumulate in a layering structure. Water molecules first fill the interior and first hydration layers before they start accumulating in the exterior region. The interior and first hydration layers become saturated for hydration levels $7<\omega<10$, in agreement with X-ray scattering experiments [36]. Note that for the least hydrated cases $(\omega=4,7)$, although the interior and first hydration layers are formed, they are not yet "full". The layering structure observed in systems of hydrated stacked phospholipid bilayers is in contrast with the formation of droplets reported for low hydrated systems of confined water in between monolayers of SDS surfactant 37.

We analyze how both water-water HBs and water-lipid HBs change as a function of $\xi$ (Fig. 3). For the completely hydrated membrane (with $\omega=34$ ), we observe that the number of water-lipid HBs in the interior of the membrane is $\simeq 1$ and decreases to zero in the first hydration layer, while water-water HBs are $\simeq 2$ in the interior, 2 or more in the first hydration layer and saturates to $\simeq 3.45$ at larger distance. The number of water-water HBs suggests that water forms files of $\gtrsim 3 \mathrm{H}$-bonded molecules deep inside the membrane.

The situation does not show qualitative changes for $\omega=20,15$ and 10 , corresponding to cases for which water completes the first hydration layer of both leaflets. Instead, for the cases with $\omega=4$ and 7 , in which there is not enough water to fully hydrate the two leaflets, we observe a qualitative difference: the number of water-water HBs decreases both inside and in the first hydration layer, and the number of water-lipid HBs increases, especially in the first hy- 


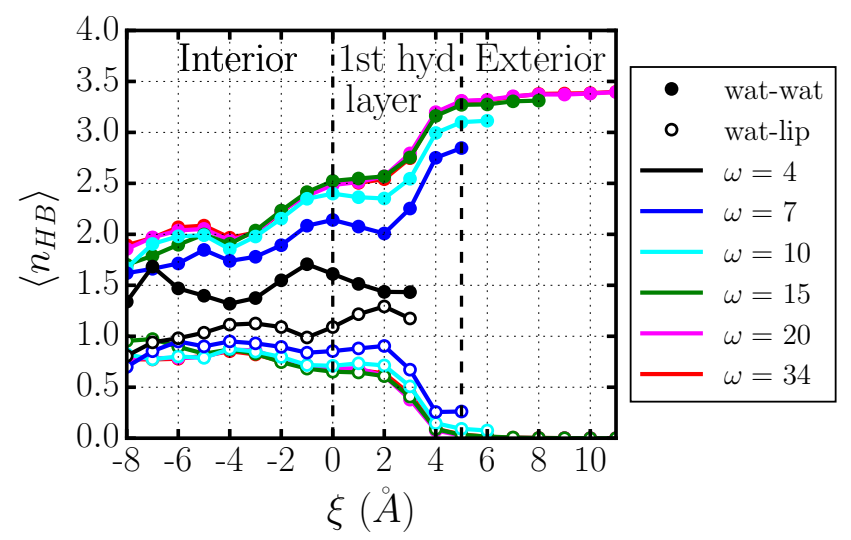

Figure 3: Average number of HBs $\left\langle n_{\mathrm{HB}}\right\rangle$ as a function of $\xi$ for the different hydrated phospholipid bilayers considered. Full circles represent HBs formed between water molecules, and empty circles the HBs formed by water molecules with selected groups of the phospholipid.

dration layer. Therefore, upon dehydration of the membrane, water partially compensates the reduced number of HBs among water molecules with an increase of HBs with lipids. This increase of adsorption of water on the polar groups of the membrane at low hydration is similar to what happens on the exposed polar residues of dry proteins [38, 39].

Overall, these observations show that at low $\omega$, water is less bonded but more adsorbed to the membrane [15. Moreover, it seems appropriate to distinguish between water H-bonded to lipids, boundhydration water and water not bonded to lipids, present at $\omega \gtrsim 7$, as unbound hydration water.

The average number of HBs, however, does not give a complete picture of the HB structure. To get a better insight we calculate the probability of a water molecule to be H-bonded with another moiety at different regions of the interface in a completely hydrated DMPC stacked membrane with $\omega=34$ (Fig. 4). The first observation is that bound hydration water accounts for $\simeq 65 \%$ of water in the interior of the membrane, because only $\simeq 35 \%$ of water molecules are non H-bonded to lipids (Fig. 4. a, central panel). This feature decreases to $\simeq 38 \%$ in the first hydration shell and vanishes for the exterior of the membrane (central 
panels of Fig. 4 $\mathrm{b}$ and c, respectively).

The local changes of the HB network are also reflected by the number of water-water HBs (left panels of Fig. 4p: in the interior of the membrane its distribution is quite symmetric with only $\simeq 42 \%$ of water forming two HBs, $\simeq 25 \%$ forming one,$\simeq 24 \%$ forming three and $\simeq 5 \%$ forming four. The distribution shifts toward higher values in the first hydration shell, with a maximum at three HBs, and in the exterior of the membrane, with a maximum between three and four HBs and a non vanishing probability of forming five (high-energy, distorted) HBs, consistent with the averages of these distributions Fig. 33.

The hydrogen bond distribution also depends on the hydration level of the membrane in cases where layers are not completely full, as shown in Fig. 5 for $\omega=4$. The fraction of bound hydration water for low hydrated membranes increases from $\simeq 18 \%$ at $\omega=34$ to $\simeq 81 \%$ at low hydration with $\omega=4$ [15], consistent with the experimental estimate of $92 \pm 7 \%$ of water H-bonded to the membrane at weak hydration [17.

We calculate, as a function of $\omega$, also the fraction of total HBs formed by water and DMPC involved in bridging, i.e. water molecules with two HBs to lipids [40, 41, 42. Such water molecules contribute to the structure and mechanical properties of the membrane [41, 42. We observe that the overall fraction of bridging HBs strongly depends on the hydration level at low values of $\omega$ and saturates at $\simeq 31 \%$ for $\omega \geq 15$ (Fig. 6 a), consistent with the case at $\omega=22.5$ previously studied [40, 41, 42]. These results confirm that for $\omega \geq 15$ the membrane interior and first hydration shell are fully hydrated (as seen in Figs. 2 and 3 and show that almost one third of the water-lipids HBs at full hydration are structural bridges, supporting our definition of bound hydration water.

In particular, our analysis reveals that a large fraction of water-lipids HBs in the interior of the membrane are bridging between two lipids (Fig. 6. b). We find that this fraction is weakly dependent on $\omega$, being always between $38 \%$ and $45 \%$. Furthermore, our calculations show that the exponential decay observed in the overall fraction is dominated by the contribution coming from the first 
(a) Interior

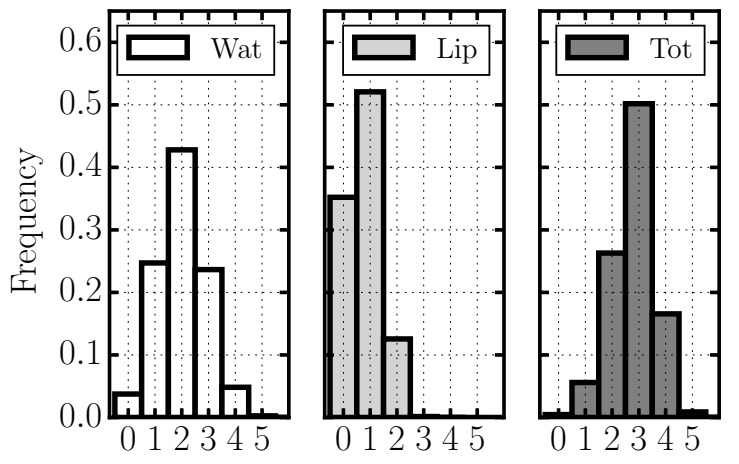

(b) 1st hydration layer

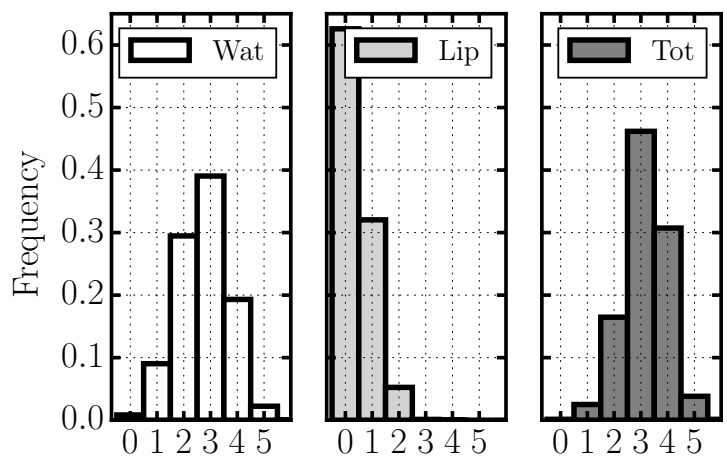

(c) Exterior

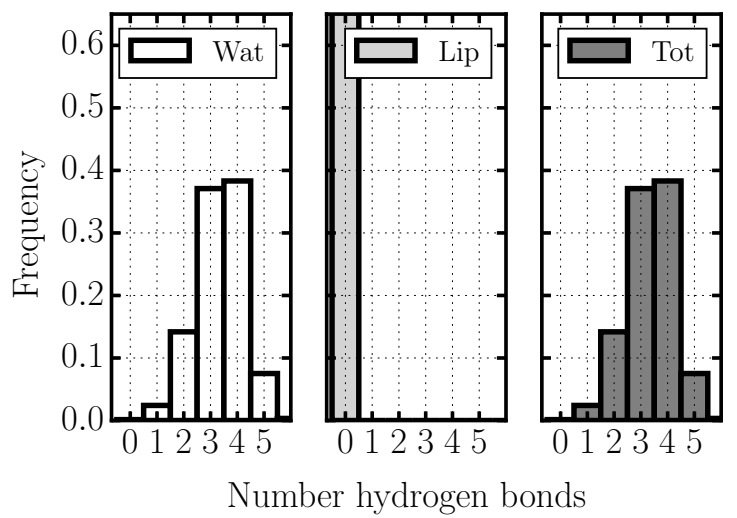

Figure 4: Normalized distribution of the number of HBs formed by a water molecule in a completely hydrated DMPC stacked membrane $(\omega=34)$ at different regions of the interface: (a) Interior $(\xi<0)$, (b) first hydration layer $(0<\xi / \AA<5)$, and (c) Exterior $(5<\xi / \AA<15)$. We symmetrize the results from the two leaflets of the phospholipid bilayer. For each case, we show the distribution of the total number of HBs and of those HBs formed with other water molecules and lipid groups. 
(a) Interior

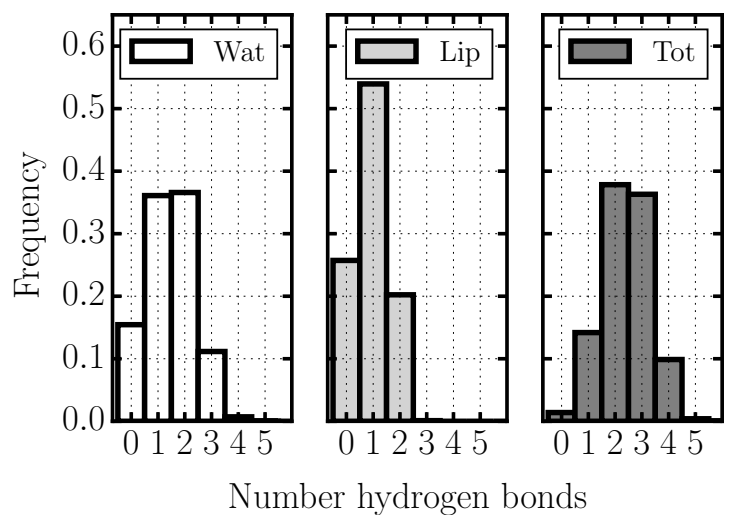

(b) 1st hydration layer

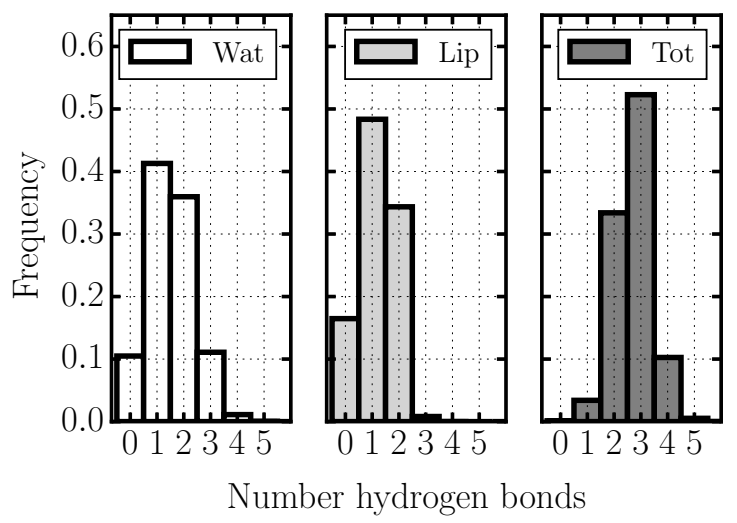

Figure 5: Normalized distribution of the number of HBs formed by a water molecule in low hydrated DMPC stacked membrane $(\omega=4)$ at different regions of the interface: (a) Interior $(\xi<0)$ and (b) first hydration layer $(0<\xi / \AA<5)$. We symmetrize the results from the two leaflets of the phospholipid bilayer. For each case, we show the distribution of the total number of HBs and of those HBs formed with other water molecules and lipid groups. 
(a)

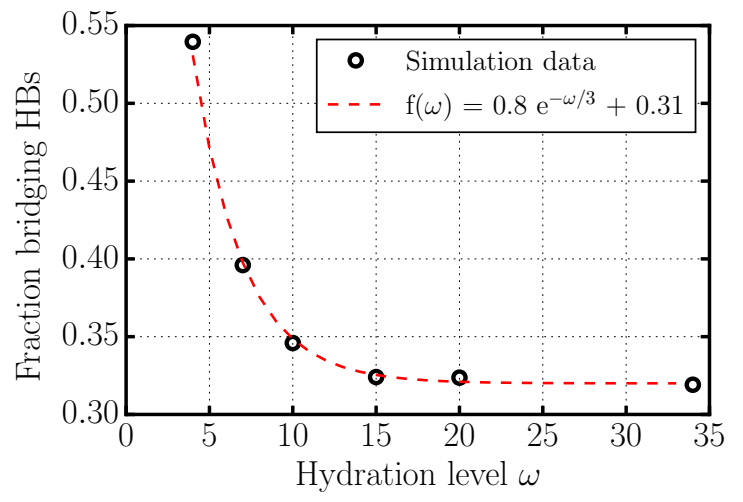

(b)

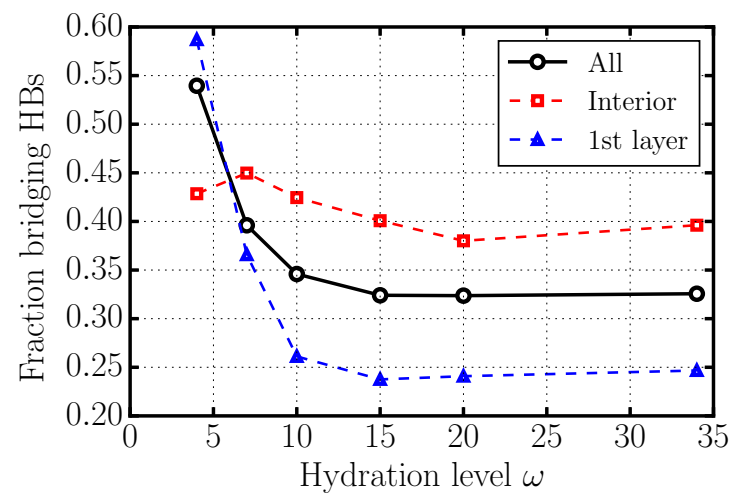

Figure 6: Fraction of bridging $\mathrm{HBs}$, with respect to the total number of water-lipids HBs, as a function of the hydration level $\omega$. (a) Overall fraction: symbols are from our simulations, line is an exponential fit with the parameters indicated in the legend. (b) Different components of the fraction of bridging HBs: for molecules in the interior of the membrane (squares), for those in the first hydration shell (triangles) and for the overall system. Lines are guides for the eyes. 
hydration shell, where for $\omega \geq 15$ approximately $1 / 4$ of the water-lipids HBs are bridging between two lipids.

The number of water-lipid HBs inside the membrane, together with the observation that water inside the membrane is bridging between different phospholipids even at the lowest hydration levels, support previous observations stating that water among the lipids have a structural function for the membrane and contribute to its stability [41, 42]. In particular, Pasenkiewicz-Gierula et al. showed that water bridges and charge pairs, between the positively and negatively charged groups of two DMPC molecules, together form an extended and dynamic network of interactions among DMPC headgroups linking $98 \%$ of all membrane phospholipids with average lifetimes of DMPC-DMPC dynamic associations of up to $1500 \mathrm{ps}$ [41].

Finally, we observe that our simulations do not show any significant fraction of unbounded water molecules in any of the regions and hydration levels $\omega$ considered (e.g., right panels of Fig. 4 and Fig. 5). However, even at low hydration level, a small amount of water is not H-bonded to lipids. For example, at $\omega=4$ we find that $\simeq 25 \%$ of water in the interior of the membrane, and $\simeq 18 \%$ in the first hydration shell, has only water-water HBs, therefore belonging to unbound hydration water.

The existence of this unbound hydration water could be the possible reason why in previous experimental works it has been hypothesized the existence of fast water in weakly hydrated phospholipid bilayers [9]. Nevertheless, as we will discuss in the following sections, our results do not support this interpretation of the experimental data, showing that both bound and unbound hydration water are slower than bulk water.

\subsection{Water translational dynamics as a function of distance from the membrane}

Next, we investigate the dynamics of water molecules as a function of their distance $\xi$ to the phospholipid membrane for the completely hydrated membrane with $\omega=34$. To study the water translational diffusive dynamics, we consider layers of $5 \AA$-width in each region, as defined from our structural analysis of the 


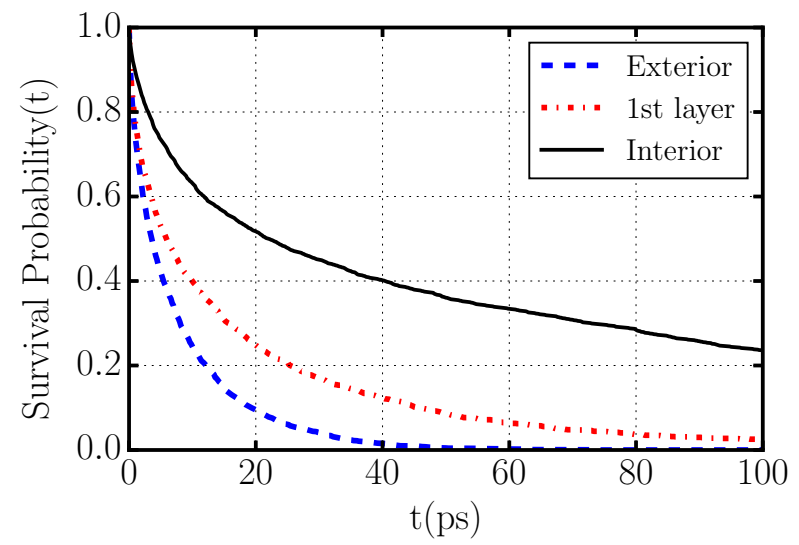

Figure 7: Survival probability for $5 \AA$-width layers in the interior, the first hydration layer and the exterior of the membrane as defined from the structural analysis for $\omega=34$.

previous paragraph, and calculate how the water survival probability changes among the regions (Fig. 7). From Eq. 22) we estimate the residence times for the different regions as $\tau_{\text {res }}^{\text {int }}=64 \mathrm{ps}, \tau_{\text {res }}^{1 \text { st }}=16 \mathrm{ps}$, and $\tau_{\text {res }}^{\text {ext }}=8 \mathrm{ps}$ for water molecules in the interior, first hydration layer and exterior region, respectively.

Once we know $\tau_{\text {res }}^{(i)}$ for each layer $i$ with $5 \AA$-width, we can correctly calculate the $\operatorname{MSD}_{\|}(t)$ of water molecules in each layer for $t<\tau_{\text {res }}^{(i)}$ (Fig. 8 a), considering layers up to the center of the system (with $\xi<20 \AA$ ). We observe that for all distances, water molecules are in the diffusive regime for $t>2 \mathrm{ps}$, with $\mathrm{MSD}_{\|}(t)$ scaling linearly with time. Furthermore, the calculations are clearly grouping in three main behaviors corresponding to the three regions, interior, first hydration layer and exterior of the membrane.

From the diffusive regime we can estimate the diffusion coefficient for each layer $D_{\|}(\xi)$ (Fig. 8 b). We find that $D_{\|}(\xi)$ increases monotonically with distance to the membrane. However, even at the largest distance from the membrane, $\simeq 18 \AA$, the in-layer diffusion coefficient does not reach the bulk value, $D^{\text {bulk }} \simeq 6$ $\mathrm{nm}^{2} / \mathrm{ns}$ at $303 \mathrm{~K}[43,44$.

In particular, we observe that, within the membrane, water has a diffusion coefficient more than 20 times smaller than bulk water, at the first hydration 
shell $D_{\|}$is $\simeq 15 \%$ than the bulk value and, at the layer centered between the two confining membranes at $\simeq 15 \AA$, the in-plane diffusion coefficient is only $\simeq 83 \%$ than the bulk value, indicating a long range effect of the interface on water translational dynamics 1

\subsection{Water rotational dynamics as a function of distance from the membrane}

Next, we calculate the rotational correlation function $C_{\mathrm{sim}}^{\mathrm{rot}}(t)$ for water molecules at the interior, exterior and first-hydration layers (Fig. 9). We can clearly identify three different behaviors depending on the local distance $\xi$ of water molecules from the membrane: one associated to the interior, one for the first hydration layer and another for the exterior of the membrane. To each region corresponds a different rotational relaxation time. In particular, for water inside the membrane we estimate the characteristic relaxation times as $\tau_{R}^{(1)}=28 \mathrm{ps}$, for the first hydration layer as $\tau_{R}^{(2)}=6.1 \mathrm{ps}$ and for the water at further distance as $\tau_{R}^{(3)}=2.4 \mathrm{ps}$.

The prediction of the model for bulk water at ambient condition is $\tau_{R} \simeq$ $1.9 \mathrm{ps}$, consistent with the value reported in the literature [45] ${ }^{2}$ Therefore, the membrane slows down the orientational dynamics of water in its interior by a factor $\simeq 15$ and in the first hydration shell by a factor $\simeq 3$. Water at the furthest distance, with $10 \AA<\xi<15 \AA$, still exhibits a $20 \%$ slower orientation relaxation than bulk water.

This result demonstrates a clear correlation between the location of water molecules within the interface and their rotational dynamics and validates the division into layers done in terms of its structural properties 9 .

\footnotetext{
${ }^{1}$ The facts that the diffusion is limited in a quasi-2-dimensional layer and that in 2 dimensions the diffusion constant is expected to be larger than in 3 dimensions, reinforce the result.

${ }^{2}$ TIP3P-water underestimates the orientational relaxation time when compared with the experiments by a factor between two and three [46].
} 

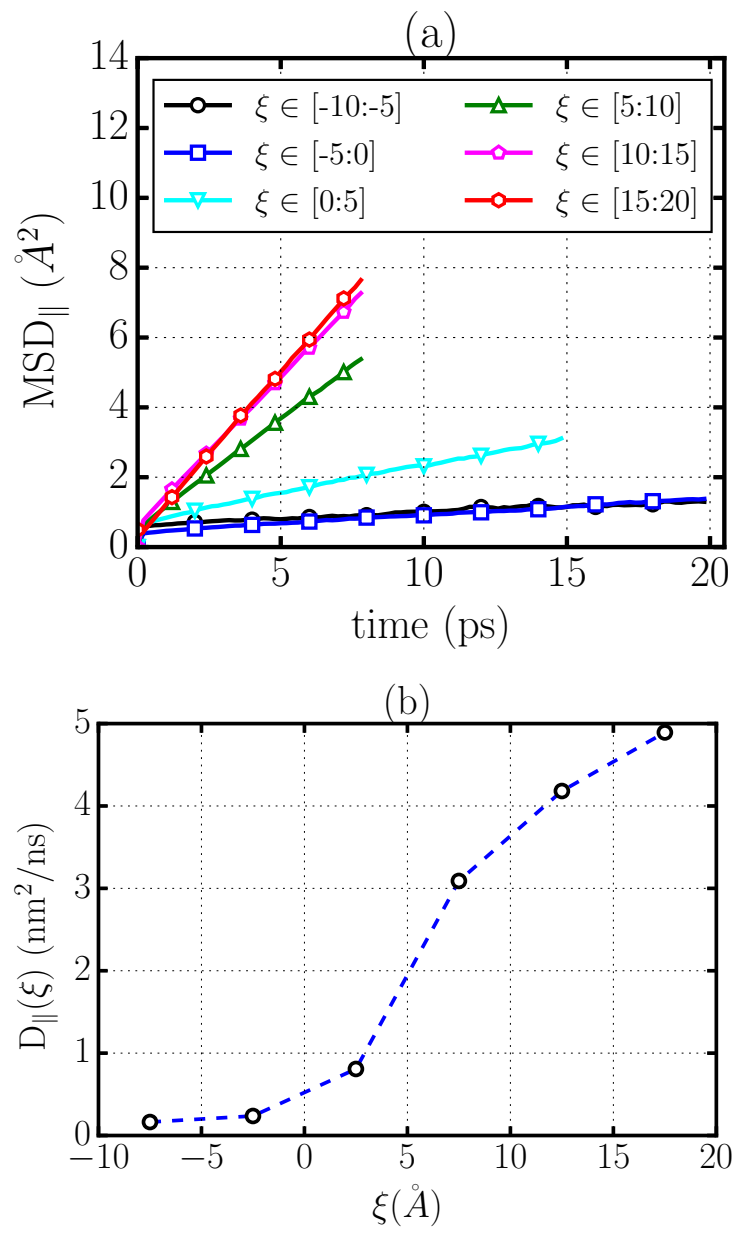

Figure 8: Translational diffusion dynamics of water molecules on planes parallel to the membrane as a function of distance $\xi$ for $\omega=34$. (a) $\operatorname{MSD}_{\|}(t)$ for water molecules with $-10 \leq \xi / \AA<-5$ (black dots), $-5 \leq \xi / \AA<0$ (blue squares), $0 \leq \xi / \AA<5$ (cyan down triangles), $5 \leq \xi / \AA ̊ 210$ (green up triangles), $10 \leq \xi / \AA<15$ (magenta pentagons), $15 \leq \xi / \AA<20$ (red hexagons). (b) Diffusion coefficient of water molecules in layers centered at $\xi$. 


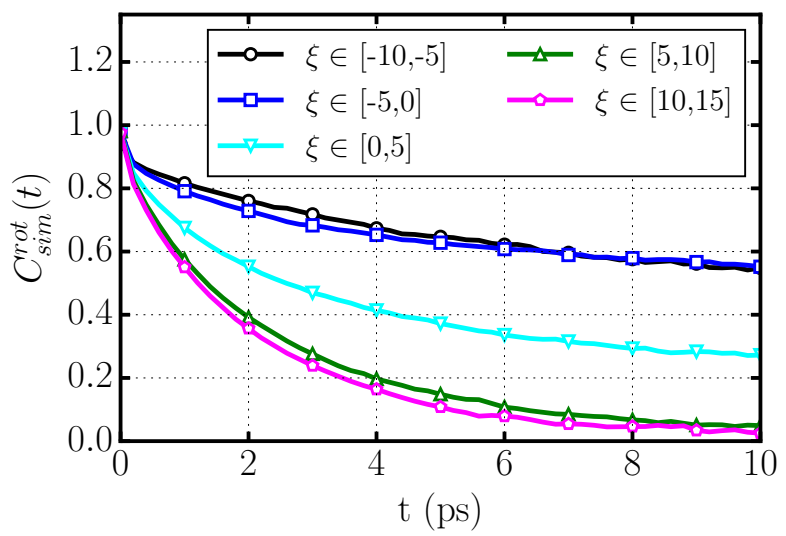

Figure 9: Dipolar rotational correlation function of water molecules as a function of distance $\xi$ from the membrane for $\omega=34$, for $-10 \leq \xi / \AA<-5$ (black dots), $-5 \leq \xi / \AA<0$ (blue squares), $0 \leq \xi / \AA<5$ (cyan down triangles), $5 \leq \xi / \AA<10$ (green up triangles), $10 \leq \xi / \AA<15$ (magenta pentagons).

\subsection{HB dynamics as a function of distance from the membrane}

The dynamical properties of liquid water are determined by its capacity of breaking and reforming HBs with other molecules. Hence, as in Ref. [15, we relate the water dynamics to its HB dynamics. While in Ref. [15 the analysis was performed averaging over all the different regions of the interface, here we calculate for the fully hydrated case with $\omega=34$ the water-water and the water-lipid $\mathrm{HB}$ correlation functions, $C_{\mathrm{HB}}^{\mathrm{w}-\mathrm{w}}(t)$ and $C_{\mathrm{HB}}^{\mathrm{w}-\text { lip }}(t)$ respectively, in each separate region: the interior, the first hydration layer and the exterior of the membrane (Fig. 10 ).

We find that the water-water HBs in the interior region of the membrane are significantly more robust than in the exterior (Fig. 10 a). Their behavior resemble what we found on average for the low hydrated cases 15] and can be explained in a similar way, based on two main reasons. One, they form longlived HBs with the lipid headgroup (Fig. 10.b), which anchors them in a fixed position for long times. Second, the density of water inside the membrane, lower than in the other two regions, suppresses the HB switching events between water 
molecules [47, 13]. In the first hydration layer, although the water density is higher than in the exterior layers (Fig. 2), water molecules form also long-lived HBs with the lipid (Fig. 10. b) and $C_{\mathrm{HB}}^{\mathrm{w}-\mathrm{w}}(t)$ decays slower than in the exterior layers.

Therefore, the slowdown of translation and rotational dynamics of water inside the membrane and in its first hydration shell is a consequence of the strong water-lipid HBs, as demonstrated by Bhide and Berkowitz in Ref. [12. This result reinforce the concept of bound hydration water as structurally and dynamically different from unbound hydration water at the membrane.

Nevertheless, the second reason of the dynamics slowdown at the membrane interface associated to the density variation, i.e. the suppression of HB switching due to the lack of available water molecules, also plays a relevant role. This can be better seen by reducing the hydration, as we discuss in the next section.

\subsection{Effect of layer filling on water dynamics}

By decreasing $\omega$, as seen in our structural analysis, we change the water filling of the layers with a strong effect on the water dynamics, as we can see by calculating the rotational correlation function for the interior and the first hydration layer of the membrane (Fig 11 ). We observe that for systems in which a certain layer is not completely filled, i.e., the cases with $\omega=4$ and 7 for the interior region and $\omega=7$ and 10 for the first hydration layer, the relaxation of the rotational correlation function is slower than for cases with higher $\omega$ and filled layers. In fact, the relaxation for all cases with higher $\omega$ coincide. This observation suggests that the lack of water molecules hinders the hydrogen bond switch slowing down the dynamics. The slower dynamics of water molecules in the inner layer and in the partially-filled first hydration layer, occurring in cases with $\omega=4$ and 7 , could be an indication of structural changes in the phospholipid headgroup due to the proximity of the two opposing leaflets.

This is consistent with our calculation for the water-water HB correlation function behavior for decreasing $\omega$ within the interior of the membrane. Indeed, for those water molecules in systems where the interior layer is not complete, 

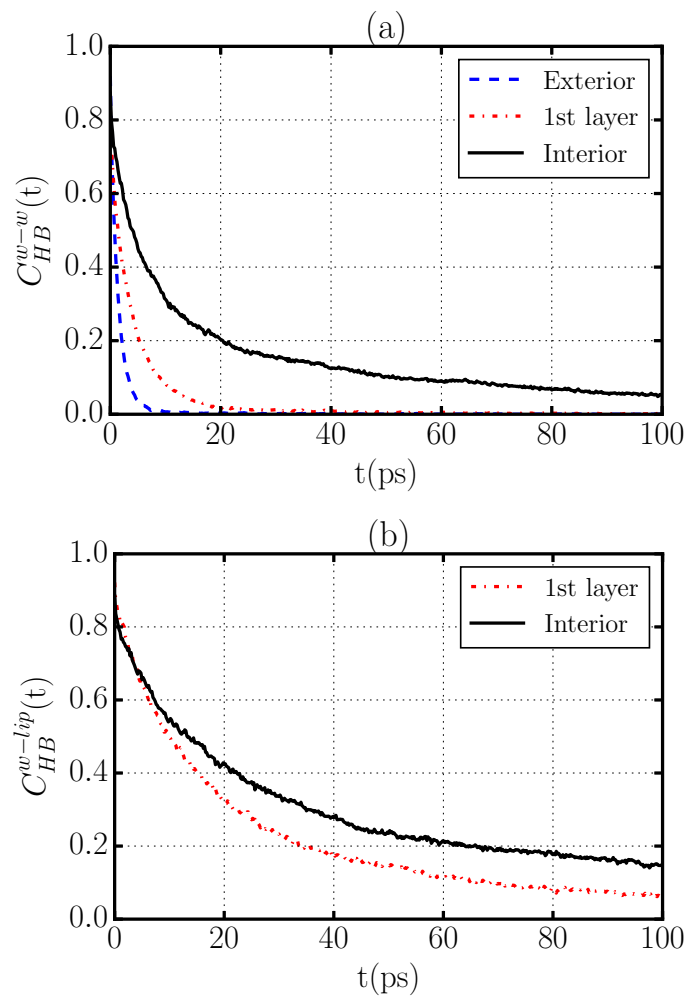

Figure 10: Relaxation with time of the correlation functions for $\omega=34$ of HBs formed by water molecules with (a) other water molecules, and (b) with selected lipid groups for water molecules in the interior, the first hydration layers and the exterior of the membrane. 

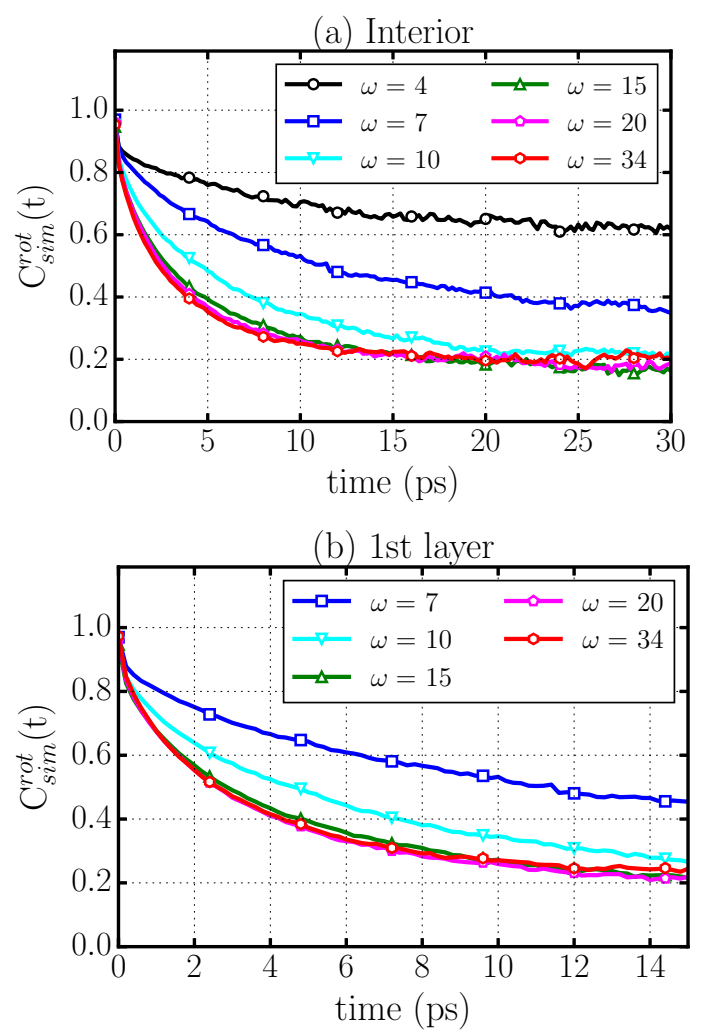

Figure 11: Dipolar rotational correlation function of water molecules in the (a) interior and (b) first hydration layers of stacked DMPC bilayers with different hydration $\omega$.

the breakage of HBs is slower (Fig. 12 a). Furthermore, it is reasonable also to conclude that the suppression of HB-switching events between lipids and water is responsible for the slower relaxation of the water-lipid HB correlation function (Fig. 10,b).

\section{Conclusions}

We consider different hydration levels $\omega$ for stacked phospholipid membranes. To filter out the smoothing effect of the fluctuation interface, we adopt a local definition of distance $\xi$ from the membrane 32 to perform our analysis of the structure and dynamics of water. 

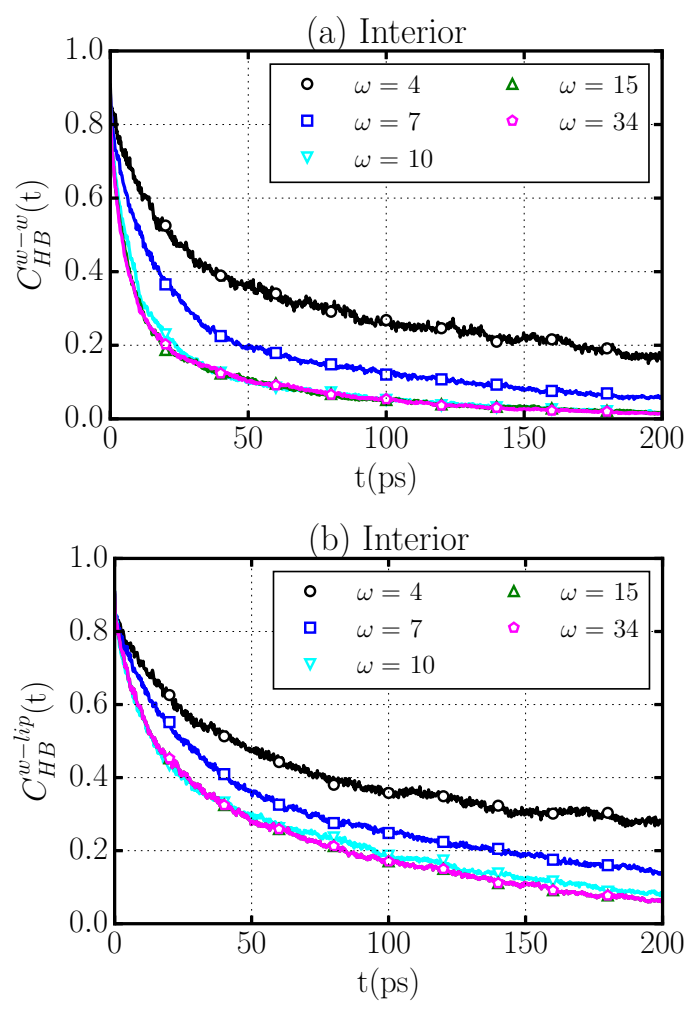

Figure 12: Relaxation with time of the correlation functions of HBs formed by water molecules with (a) other water molecules, and (b) with selected lipid groups for water molecules in the interior of the membrane for different hydration levels $\omega=4$ (black dots), 7 (blue squares), 10 (cyan triangle down), 15 (green triangle up), and 34 (magenta pentagon). 
We show that, as the hydration of the membrane increases, water accumulates in a layering fashion, starting from the interior $(\xi<0)$ and the first hydration shell $(0<\xi / \AA<5)$ of the membrane. These bounded water molecules form strong $\mathrm{HBs}$ with the lipids and become part of the membrane, playing a possible role in stabilizing the phospholipid self-assembly with structural water bridges between lipids and persistent HBs. Translation and rotational dynamics of bound hydration water is almost frozen, with a diffusion coefficient 20 times smaller, and a rotational dynamics 15 times slower, than in bulk.

Although at small hydration levels the majority of water is H-bonded to the membrane, part of it (e.g., $\simeq 20 \%$ at $\omega=4$ ) is not, being unbound hydration water. The amount of unbound hydration water rapidly increases once the level of bound hydration water saturates, which occurs for hydration levels $\omega \gtrsim 15$, accumulating in the exterior region with $\xi>5 \AA$.

Unbound hydration water is much more mobile than water bound to the membrane, forming only water-water HBs. Nevertheless, it is still slower than bulk water, with an evident slowing-down effect of the interface even at $\xi \simeq$ $17.5 \AA$, the largest possible distance from membranes in our geometry. We show that the intensity of the effect depends on $\xi$ : e.g., the water diffusion coefficient in a layer at $5 \AA$ from the membrane is $85 \%$ smaller than in the bulk, and at $\xi \simeq 17.5 \AA$ it is still $17 \%$ smaller than in bulk. We find a similar effect for the rotational dynamics with respect to bulk, with a slowing-down factor of 3 in the first hydration shell and a $20 \%$ slower at $\xi \simeq 15 \AA$.

In both translational and rotational dynamics we find that the largest variation of the dynamics occurs across the first hydration layer $(0<\xi / \AA<5)$ and the beginning of the exterior region $(5<\xi / \AA<10)$. Therefore, our results show that the dynamics has a maximum variation at $\simeq 5 \AA$, marking an "interface" between the first hydration shell, partially made of bound hydration water, and the exterior at $\xi>5 \AA$, entirely made of unbound hydration water, reinforcing the qualitative difference between the two kinds of water.

Our structural analysis and detail study of the HB dynamics allows us to show how all these features depend on the different HBs that water molecules 
forms in the different regions of the interface. The results presented here might be relevant to understand the slowdown of biological activity upon dehydration. They are part of our efforts to develop a multiscale approach to gain access to large scale and long time simulations of nanobio systems [48] where all the components are considered, at least at coarse-grained level, including water [49, 50], proteins [51, 52, 53, proteins interfaces [54], protein-protein interactions [55, 56] and membranes.

\section{Acknowledgements}

We are thankful to Fabio Leoni and Fausto Martelli for helpful discussions. We acknowledge the support of Spanish MINECO grant FIS2012-31025 and FIS2015-66879-C2-2-P.

\section{References}

[1] I. W. Hamley, Introduction to Soft Matter, John Wiley and sons, West Sussex, England, 2007.

[2] M. L. Berkowitz, D. L. Bostick, S. Pandit, Aqueous solutions next to phospholipid membrane surfaces: insights from simulations, Chemical reviews 106 (4) (2006) 1527-1539.

[3] S. R. Wassall, Pulsed field gradient-spin echo nmr studies of water diffusion in a phospholipid model membrane, Biophysical Journal 71 (1996) 27242732 .

[4] F. Volke, S. Eisenblätter, J. Galle, G. Klose, Dynamic properties of water at phosphatidylcholine lipid-bilayer surfaces as seen by deuterium and pulsed field gradient proton nmr, Chemistry and physics of lipids 70 (2) (1994) $121-131$.

[5] S. König, E. Sackmann, D. Richter, R. Zorn, C. Carlile, T. Bayerl, Molecular dynamics of water in oriented dppc multilayers studied by quasielastic 
neutron scattering and deuterium-nuclear magnetic resonance relaxation, The Journal of chemical physics 100 (4) (1994) 3307-3316.

[6] X. Chen, W. Hua, Z. Huang, H. C. Allen, Interfacial water structure associated with phospholipid membranes studied by phase-sensitive vibrational sum frequency generation spectroscopy, Journal of the American Chemical Society 132 (32) (2010) 11336-11342.

[7] H. Binder, The molecular architecture of lipid membranes - new insights from hydration-tuning infrared linear dichroism spectroscopy, Applied Spectroscopy Reviews 38 (1) (2003) 15-69. arXiv:https://doi.org/10. 1081/ASR-120017480, doi:10.1081/ASR-120017480. URL https://doi.org/10.1081/ASR-120017480

[8] W. Zhao, D. E. Moilanen, E. E. Fenn, M. D. Fayer, Water at the surfaces of aligned phospholipid multibilayer model membranes probed with ultrafast vibrational spectroscopy, Journal of the American Chemical Society 130 (42) (2008) 13927-13937, pMID: 18823116. arXiv:http://dx.doi. org/10.1021/ja803252y, doi:10.1021/ja803252y URL http://dx.doi.org/10.1021/ja803252y

[9] K. J. Tielrooij, D. Paparo, L. Piatkowski, H. J. Bakker, M. Bonn, Dielectric relaxation dynamics of water in model membranes probed by terahertz spectroscopy, Biophysical Journal 97 (2009) 2848-2492.

[10] M. Trapp, T. Gutberlet, F. Juranyi, T. Unruh, B. Demé, M. Tehei, J. Peters, Hydration dependent studies of highly aligned multilayer lipid membranes by neutron scattering, The Journal of chemical physics 133 (16) (2010) 164505.

[11] R. W. Pastor, Molecular dynamics and monte carlo simulations of lipid bilayers, Current Opinion in Structural Biology 4 (4) (1994) 486-492.

[12] S. Y. Bhide, M. L. Berkowitz, Structure and dynamics of water at the inter- 
face with phospholipid bilayers, The Journal of chemical physics 123 (22) (2005) 224702.

[13] Z. Zhang, M. L. Berkowitz, Orientational dynamics of water in phospholipid bilayers with different hydration levels, The Journal of Physical Chemistry B 113 (21) (2009) 7676-7680.

[14] S. Gruenbaum, J. Skinner, Vibrational spectroscopy of water in hydrated lipid multi-bilayers. i. infrared spectra and ultrafast pump-probe observables, The Journal of chemical physics 135 (7) (2011) 075101.

[15] C. Calero, H. E. Stanley, G. Franzese, Structural interpretation of the large slowdown of water dynamics at stacked phospholipid membranes for decreasing hydration level: All-atom molecular dynamics, Materials 9 (5) (2016) 319. doi:10.3390/ma9050319

URL http://www .mdpi .com/1996-1944/9/5/319

[16] F. Martelli, H.-Y. Ko, C. C. Borallo, G. Franzese, Structural properties of water confined by phospholipid membranes, Frontiers of Physics 13 (1) (2018) 136801.

[17] V. V. Volkov, D. J. Palmer, R. Righini, Distinct water species confined at the interface of a phospholipid membrane, Phys. Rev. Lett. 99 (2007) 078302. doi:10.1103/PhysRevLett.99.078302.

URL http://link.aps.org/doi/10.1103/PhysRevLett.99.078302

[18] J.F.Nagle, R.Zhang, S.Tristam-Nagle, H.I.Petrache, R.M.Suter, Biophysical Journal 70 (1996) 1419.

[19] J. C. Phillips, R. Braun, W. Wang, J. Gumbart, E. Tajkhorshid, E. Villa, C. Chipot, R. D. Skeel, L. Kalé, K. Schulten, Scalable molecular dynamics with NAMD, J. Comput. Chem. 26 (16) (2005) 1781-1802. doi:10.1002/ jcc.20289.

[20] J. B. Klauda, R. M. Venable, J. A. Freites, J. W. O'Connor, D. J. Tobias, C. Mondragon-Ramirez, I. Vorobyov, A. D. MacKerell, R. W. Pastor, 
Update of the CHARMM All-Atom Additive Force Field for Lipids: Validation on Six Lipid Types, J. Phys. Chem. B 114 (23) (2010) 7830-7843. doi:10.1021/jp101759q.

[21] J. B. Lim, B. Rogaski, J. B. Klauda, Update of the cholesterol force field parameters in CHARMM, J. Phys. Chem. B 116 (1) (2012) 203-210. doi: $10.1021 / j p 207925 \mathrm{~m}$

[22] W. L. Jorgensen, J. Chandrasekhar, J. D. Madura, R. W. Impey, M. L. Klein, Comparison of Simple Potential Functions for Simulating Liquid Water, J. Chem. Phys. 79 (2) (1983) 926-935. doi:10.1063/1.445869. URL http://scitation.aip.org/content/aip/journal/jcp/79/2/10. $1063 / 1.445869$

[23] J. A. D. MacKerell, D. Bashford, M. Bellott, J. R. L. Dunbrack, J. D. Evanseck, M. J. Field, S. Fischer, J. Gao, H. Guo, S. Ha, D. JosephMcCarthy, L. Kuchnir, K. Kuczera, F. T. K. Lau, C. Mattos, S. Michnick, T. Ngo, D. T. Nguyen, B. Prodhom, W. E. Reiher, B. Roux, M. Schlenkrich, J. C. Smith, R. Stote, J. Straub, M. Watanabe, J. Wiórkiewicz-Kuczera, D. Yin, M. Karplus, All-atom empirical potential for molecular modeling and dynamics studies of proteins, The Journal of Physical Chemistry B 102 (18) (1998) 3586-3616. doi:10.1021/jp973084f

[24] U.Essmann, L.Perera, M.L.Berkowitz, T.Darden, H.Lee, L.G.Pedersen, J. Chem. Phys. 103 (1995) 8577.

[25] H.J.C.Berendsen, J.P.M.Postma, W. Gunsteren, A.DiNola, J.R.Haak, J.Phys.Chem. 81 (1984) 3684.

[26] S. Feller, Y. Zhang, R. Pastor, B. Brooks, J.Phys.Chem. 103 (1995) 4613.

[27] A. Aksimentiev, K. Schulten, Imaging $\alpha$-hemolysin with molecular dynamics: ionic conductance, osmotic permeability, and the electrostatic potential map, Biophysical journal 88 (6) (2005) 3745-3761. 
[28] S. L. Duncan, R. G. Larson, Comparing experimental and simulated pressure-area isotherms for dppc, Biophysical Journal 94 (8) (2008) 2965-2986. doi:https://doi.org/10.1529/biophysj.107.114215.

URL http://www.sciencedirect.com/science/article/pii/ S0006349508704557

[29] S. E. Feller, R. W. Pastor, Constant surface tension simulations of lipid bilayers: The sensitivity of surface areas and compressibilities, The Journal of Chemical Physics 111 (3) (1999) 1281-1287. doi:10.1063/1.479313. URL https://doi.org/10.1063/1.479313

[30] J. Yang, C. Calero, J. Martí, Diffusion and spectroscopy of water and lipids in fully hydrated dimyristoylphosphatidylcholine bilayer membranes J. Chem. Phys. 140 (10) (2014) 104901. doi:10.1063/1.4867385

URL http://www.ncbi.nlm.nih.gov/pubmed/24628199

[31] J. Fitter, R. E. Lechner, N. A. Dencher, Interactions of hydration water and biological membranes studied by neutron scattering, The Journal of Physical Chemistry B 103 (38) (1999) 8036-8050. doi:10.1021/jp9912410.

[32] S. A. Pandit, D. Bostick, M. L. Berkowitz, An algorithm to describe molecular scale rugged surfaces and its application to the study of a water/lipid bilayer interface, The Journal of chemical physics 119 (4) (2003) 2199-2205.

[33] C. F. Lopez, S. O. Nielsen, M. L. Klein, P. B. Moore, Hydrogen bonding structure and dynamics of water at the dimyristoylphosphatidylcholine lipid bilayer surface from a molecular dynamics simulation, The Journal of Physical Chemistry B 108 (21) (2004) 6603-6610.

[34] A. M. Smondyrev, M. L. Berkowitz, Molecular dynamics simulation of dppc bilayer in dmso, Biophysical Journal 76 (5) (1999) 2472-2478. doi:https://doi.org/10.1016/S0006-3495(99)77402-3.

URL http://www.sciencedirect.com/science/article/pii/ S0006349599774023 
[35] F. Leoni, G. Franzese, Structural behavior and dynamics of an anomalous fluid between attractive and repulsive walls: Templating, molding, and superdiffusion, The Journal of chemical physics 141 (17) (2014) 174501.

[36] N. Kučerka, Y. Liu, N. Chu, H. I. Petrache, S. Tristram-Nagle, J. F. Nagle, Structure of fully hydrated fluid phase dmpc and dlpc lipid bilayers using $\mathrm{x}$-ray scattering from oriented multilamellar arrays and from unilamellar vesicles, Biophysical journal 88 (4) (2005) 2626-2637.

[37] F. Bresme, E. Chacón, H. Martínez, P. Tarazona, Adhesive transitions in newton black films: A computer simulation study, The Journal of chemical physics 134 (21) (2011) 214701.

[38] S. E. Pagnotta, R. Gargana, F. Bruni, A. Bocedi, Glassy behavior of a percolative water-protein system, Physical Review E 71 (3) (2005) 031506. doi:10.1103/PhysRevE.71.031506

[39] M. G. Mazza, K. Stokely, S. E. Pagnotta, F. Bruni, H. E. Stanley, G. Franzese, More than one dynamic crossover in protein hydration water, Proceedings of the National Academy of Sciences 108 (50) (2011) 1987319878.

[40] M. Pasenkiewicz-Gierula, Y. Takaoka, H. Miyagawa, K. Kitamura, A. Kusumi, Hydrogen bonding of water to phosphatidylcholine in the membrane as studied by a molecular dynamics simulation: Location, geometry, and lipidlipid bridging via hydrogen-bonded water, The Journal of Physical Chemistry A 101 (20) (1997) 3677-3691. doi:10.1021/jp962099v.

URL https://doi.org/10.1021/jp962099v

[41] M. Pasenkiewicz-Gierula, Y. Takaoka, H. Miyagawa, K. Kitamura, A. Kusumi, Charge pairing of headgroups in phosphatidylcholine membranes: A molecular dynamics simulation study, Biophysical Journal 76 (3) (1999) 1228-1240. doi:10.1016/S0006-3495(99) 77286-3.

URL https://doi .org/10.1016/S0006-3495(99)77286-3 
[42] C. F. Lopez, S. O. Nielsen, M. L. Klein, P. B. Moore, Hydrogen bonding structure and dynamics of water at the dimyristoylphosphatidylcholine lipid bilayer surface from a molecular dynamics simulation, The Journal of Physical Chemistry B 108 (21) (2004) 6603-6610. doi:10.1021/ jp037618q.

URL https://doi.org/10.1021/jp037618q

[43] C. Vega, J. L. Abascal, M. Conde, J. Aragones, What ice can teach us about water interactions: a critical comparison of the performance of different water models, Faraday discussions 141 (2009) 251-276.

[44] P. Mark, L. Nilsson, Structure and dynamics of the tip3p, spc, and spc/e water models at $298 \mathrm{k}$. The Journal of Physical Chemistry A 105 (43) (2001) 9954-9960. doi:10.1021/jp003020w.

URL https://doi.org/10.1021/jp003020w

[45] D. van der Spoel, P. J. van Maaren, H. J. C. Berendsen, A systematic study of water models for molecular simulation: Derivation of water models optimized for use with a reaction field, The Journal of Chemical Physics 108 (24) (1998) 10220-10230. doi:10.1063/1.476482.

URL https://doi.org/10.1063/1.476482

[46] C. Vega, J. L. F. Abascal, Simulating water with rigid non-polarizable models: a general perspective, Physical Chemistry Chemical Physics 13 (44) (2011) 19663-19688. doi:10.1039/C1CP22168J

URL http://dx.doi.org/10.1039/C1CP22168J

[47] D. Laage, J. T. Hynes, A molecular jump mechanism of water reorientation, Science 311 (5762) (2006) 832-835.

[48] O. Vilanova, V. Bianco, G. Franzese, Multi-Scale Approach for SelfAssembly and Protein Folding, Springer International Publishing, Cham, 2017, pp. 107-128. doi:10.1007/978-3-319-71578-0\{\_\}5.

URL https : //doi .org/10.1007/978-3-319-71578-0_5 
[49] F. de los Santos, G. Franzese, Understanding diffusion and density anomaly in a coarse-grained model for water confined between hydrophobic walls, The Journal of Physical Chemistry Bdoi:10.1021/jp206197t.

URL http://pubs . acs . org/doi/abs/10.1021/jp206197t

[50] V. Bianco, G. Franzese, Critical behavior of a water monolayer under hydrophobic confinement, Sci. Rep. 4.

URL http://dx.doi.org/10.1038/srep04440

[51] V. Bianco, S. Iskrov, G. Franzese, Understanding the role of hydrogen bonds in water dynamics and protein stability, Journal of Biological Physics 38 (1) (2012-01-01) 27-48. doi:10.1007/s10867-011-9235-7.

URL http://dx.doi.org/10.1007/s10867-011-9235-7

[52] V. Bianco, G. Franzese, Contribution of water to pressure and cold denaturation of proteins, Physical Review Letters 115 (10) (2015) 108101-.

URL http://link .aps .org/doi/10.1103/PhysRevLett.115.108101

[53] V. Bianco, G. Franzese, C. Dellago, I. Coluzza, Role of water in the selection of stable proteins at ambient and extreme thermodynamic conditions, Phys.

Rev. X 7 (2017) 021047. doi:10.1103/PhysRevX.7.021047.

URL https://link.aps.org/doi/10.1103/PhysRevX.7.021047

[54] P. Vilaseca, K. A. Dawson, G. Franzese, Understanding and modulating the competitive surface-adsorption of proteins through coarse-grained molecular dynamics simulations, Soft Matter (2013) -.

URL http://dx.doi .org/10.1039/C3SM50220A

[55] J. Wedekind, L. Xu, S. V. Buldyrev, H. E. Stanley, D. Reguera, G. Franzese, Optimization of crystal nucleation close to a metastable fluid-fluid phase transition, Sci. Rep. 5.

URL http://dx.doi.org/10.1038/srep11260

[56] O. Vilanova, J. J. Mittag, P. M. Kelly, S. Milani, K. A. Dawson, J. O. Rädler, G. Franzese, Understanding the kinetics of protein-nanoparticle 
corona formation, ACS Nano 10 (12) (2016) 10842-10850. doi:10.1021/

acsnano.6b04858.

URL http://dx.doi.org/10.1021/acsnano.6b04858 